\title{
Multiplatform Biomarker Discovery for Bladder Cancer Recurrence Diagnosis
}

\author{
Marine De Paoli, ${ }^{1}$ Selma Gogalic, ${ }^{2}$ Ursula Sauer, ${ }^{2}$ Claudia Preininger, ${ }^{2}$ Hardev Pandha, ${ }^{3}$ \\ Guy Simpson, ${ }^{3}$ Andras Horvath, ${ }^{3}$ and Christophe Marquette ${ }^{4}$ \\ ${ }^{1}$ AXO Science SAS, 34 rue du Mail, 69004 Lyon, France \\ ${ }^{2}$ AIT Austrian Institute of Technology GmbH, Konrad-Lorenz-Straße 24, 3430 Tulln, Austria \\ ${ }^{3}$ Department of Oncology, Faculty of Health and Medical Sciences, University of Surrey, Leggett Building, Surrey GU2 7WG, UK \\ ${ }^{4}$ Université de Lyon, Université Lyon 1, CNRS, INSA, CPE-Lyon, ICBMS, UMR 5246, No. 43, boulevard du 11 Novembre 1918, \\ 69622 Villeurbanne Cedex, France
}

Correspondence should be addressed to Christophe Marquette; christophe.marquette@univ-lyon1.fr

Received 17 May 2016; Accepted 27 July 2016

Academic Editor: Roland Seiler

Copyright (C) 2016 Marine De Paoli et al. This is an open access article distributed under the Creative Commons Attribution License, which permits unrestricted use, distribution, and reproduction in any medium, provided the original work is properly cited.

Purpose. Nonmuscle invasive bladder cancer (BCa) has a high recurrence rate requiring lifelong surveillance. Urinary biomarkers are promising as simple alternatives to cystoscopy for the diagnosis of recurrent bladder cancer. However, no single marker can achieve the required accuracy. The purpose of this study was to select a multiparameter panel, comprising urinary biomarkers and clinical parameters, for BCa recurrence diagnosis. Experimental Design. Candidate biomarkers were measured in urine samples of $\mathrm{BCa}$ patients with recurrence and BCa patients without recurrence. A multiplatform strategy was used for marker quantification comprising a multiplexed microarray and an automated platform for ELISA analysis. A multivariate statistical analysis combined the results from both platforms with the collected clinical data. Results. The best performing combination of biomarkers and clinical parameters achieved an AUC value of 0.91, showing better performance than individual parameters. This panel comprises six biomarkers (cadherin-1, IL-8, ErbB2, IL-6, EN2, and VEGF-A) and three clinical parameters (number of past recurrences, number of BCG therapies, and stage at time of diagnosis). Conclusions. The multiparameter panel could be a useful noninvasive tool for $\mathrm{BCa}$ surveillance and potentially impact the clinical management of this disease. Validation of results in an independent cohort is warranted.

\section{Introduction}

Bladder cancer is the ninth most common cancer worldwide and the seventh most common cancer in men with a worldwide age-standardized rate of 9.0 per 100000 men [1]. The majority of newly diagnosed cases are nonmuscle invasive disease (BCa) with $75 \%$ to $85 \%$ of patients presenting tumors confined to the mucosa or submucosa (Ta, carcinoma in situ (CIS), or T1 tumors) [2, 3]. Despite the good prognosis of such tumors, there is a tendency for recurrence after initial treatment. The probability of recurrence within 5 years ranges from $30 \%$ to $80 \%$ and $10 \%$ to $30 \%$ of these cases will progress to muscle invasive disease [3-5].
Follow-up is thus an essential aspect of BCa patient management and includes ongoing monitoring for recurrence detection. Cystoscopy and urinary cytology are defined as the gold standard methods for both diagnosis and surveillance of BCa [2]. Cystoscopy is highly sensitive but is still associated with a significant false negative rate. Moreover, as a costly, invasive, and uncomfortable procedure, it contributes to the economic and psychological burden of $\mathrm{BCa}[6,7]$. Urinary cytology has a higher specificity ranging from $85 \%$ to $100 \%$ and a high sensitivity in high-grade tumors but it lacks sensitivity in low-grade tumors $[2,8]$.

The management of patients with primary $\mathrm{BCa}$ diagnosis and postsurgical surveillance could greatly benefit from 


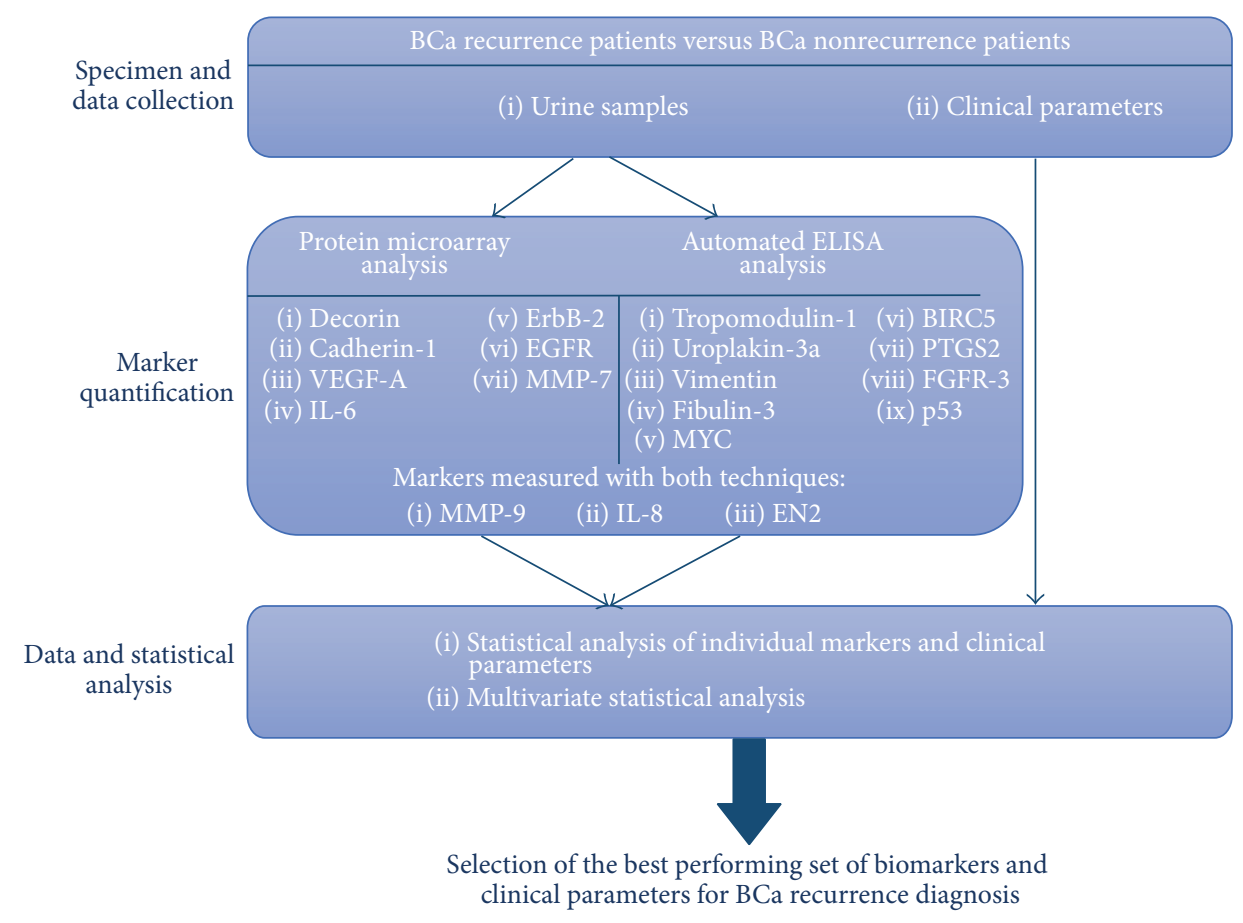

FIGURE 1: Discovery study design.

new, noninvasive methods with improved sensitivity and specificity. Urinary products of cancer growth or metabolism are highly relevant, easy to obtain, and suitable for $\mathrm{BCa}$ screening in these contexts. Urinary tests for diagnosis and detecting recurrence have already been developed, including FDA-approved BTA assays (BTA TRAK ${ }^{\circledR}$ and BTA stat ${ }^{\circledR}$ from Polymedco) as well as the Alere NMP22 ${ }^{\circledR}$ BladderChek $^{\circledR}$ Test which are used for the diagnosis and monitoring of $\mathrm{BCa}$ in conjunction with standard diagnostic procedures. They yield improved sensitivity (up to 89\%) compared to urinary cytology which has a median sensitivity of only $35 \%$. However, benign urological conditions tend to influence the specificity of these tests. They show a lower specificity than urinary cytology: the median specificity of BTA TRAK, BTA stat, and NMP22 is, respectively, $66 \%, 73 \%$, and $73 \%$ whereas urinary cytology has a median specificity of 94\% [8-10]. A recent review by van Rhijn et al. assessing the performance of 18 markers showed that urinary markers generally have a higher sensitivity but a lower specificity than urinary cytology [8]. In the context of $\mathrm{BCa}$ surveillance, the review also evaluated marker performance with regard to the detection of recurrent bladder cancer and found a lower sensitivity for most markers compared to their performance for primary disease detection. Thus, single markers are not currently suitable for incorporation into any clinical surveillance protocol to allow patients to undergo less frequent cystoscopic evaluations.

The ideal urinary test should show good performance in both sensitivity and specificity. As this is clearly not possible with single markers, combining several markers in a multiplexed assay might provide a solution for optimizing a $\mathrm{BCa}$ recurrence detection test.
A first (pilot) study was conducted by our group to identify a biomarker candidate set with potential clinical utility in BCa. The selection was made on the basis of a molecular disease model for BCa. The candidate markers were then evaluated in urine samples for their measurability and detectability in urine as well as their selectivity for BCa. This pilot study led to the definition of a five-biomarker panel (IL-8, MMP-9, VEGF-A, PTGS2, and EN2) which showed a better overall performance compared to individual markers [11]. As this study compared a group of BCa patients to a group of healthy donors, it only investigated the diagnostic aspect of de novo $\mathrm{BCa}$.

In this report, we describe a second (discovery) study which aims at selecting a marker panel for $\mathrm{BCa}$ recurrence diagnosis. Biomarkers selected in the pilot study as well as other relevant candidate markers of the molecular disease model were evaluated in urine samples of BCa patients with recurrence and $\mathrm{BCa}$ patients without recurrence. Marker measurement was performed on two different platforms: a multiplexed microarray (BCa chip) and an automated platform for 96-well plate ELISA analysis. Results from both platforms were combined with clinical parameters for a multivariate statistical analysis allowing the selection of a panel of biomarkers and clinical parameters for the diagnosis of $\mathrm{BCa}$ recurrence.

\section{Materials and Methods}

2.1. Study Design. The study design is described in Figure 1. We had access to a biobank holding 80 samples from $\mathrm{BCa}$ patients. In total, 19 markers were measured. Two analysis platforms were used for the marker measurements and the 
results were included in a statistical analysis with the patients' clinical parameters. Each study step is further described in the sections below.

2.2. Specimen and Data Collection. The selection of patients was based on the following inclusion criteria: the patient was diagnosed with $\mathrm{BCa}$ (cystoscopic and histological evidence of $\mathrm{BCa}$ ), the patient was treated for BCa prior to the sample collection visit, and the patient has no history of muscle invasive $\mathrm{BCa}$. Recurrence-negative patients are defined as showing no cystoscopic or histological evidence of BCa during monitoring after treatment of de novo $\mathrm{BCa}$. Recurrence-positive patients are defined as showing cystoscopic and histological evidence of BCa during monitoring after treatment of de novo BCa.

First pass urines were collected from the selected bladder cancer patients according to a standard operating procedure and were spun at $150 \mathrm{~g}$ for 10 minutes. The supernatant was aliquoted into $1 \mathrm{~mL}$ samples and frozen and stored at $-80^{\circ} \mathrm{C}$. All individuals gave informed consent for sample donation and the collection was approved by local ethical committee (ref. 3/LO/0739).

The samples were collected during a monitoring visit. For all samples, at least two monitoring visits are available: one for the first diagnosis and one for the point in time the sample was collected. Up to six additional visits between first diagnosis and time of sample and up to four visits after sample collection have been recorded. During the monitoring visits, the following parameters were collected: gender, age, smoking status, date, grade, stage, recurrence, TURBT (transurethral resection of the bladder tumor), and drug treatment (name and start date).

Further clinical data was collected for the statistical analysis and parameter performance regarding BCa recurrence diagnosis. Thus, the clinical parameter set used includes the following parameters: gender, age at time of diagnosis (age.diagnosis), age at time of sample collection (age.sample), time between the first diagnosis and sample collection (months.diagnosis2sample), number of past recurrences between the first diagnosis and time of sample collection (no.past.recurrences), number of bacillus CalmetteGuerin (BCG) therapies between the first diagnosis and time of sample collection (BCG.therapy), number of mitomycin therapies between the first diagnosis and time of sample collection (mitomycin.therapy), and number of transurethral resections of the bladder tumor between the first diagnosis and time of sample collection (no.past.TURBTs).

According to availability of the full set of clinical phenotype parameters and availability of valid biomarker readouts, 45 samples from the initial biobank of 80 samples could be included in the analysis, reflecting 27 patients being recurrence negative and 18 patients being recurrence positive.

2.3. Marker Quantification. Marker quantification was performed on two separate platforms. The first platform, a protein microarray (BCa chip), was used to measure the urinary levels of 10 markers, decorin, VEGF-A, cadherin1, IL-6, ErbB2, EGFR, MMP-7, MMP-9, IL-8, and EN2, while the second platform, an automated platform for 96well plate ELISA analysis, was used for the measurements of the following 12 markers: PTGS2, FGFR-3, uroplakin3a, vimentin, MYC, tropomodulin-1, BIRC5, fibulin-3, p53, MMP-9, IL-8, and EN2. Three markers (MMP-9, IL-8, and EN2) were thus measured with both methods in order to evaluate the concordance of the two analytical platforms.

\subsubsection{BCa Chip}

(1) Materials and Reagents. ARChip Epoxy (EP 02799374; US 10/490543) was used as assay platform. Recombinant human VEGF-A (293-VE-050), human VEGF-A antibody (MAB293) and human VEGF-A biotinylated antibody (BAF293), recombinant human MMP-7 (907-MP010), human MMP-7 antibody (clone 111439, MAB9072), human MMP-7 biotinylated antibody (BAF907), recombinant human MMP-9 (CF, 911-MP-010), human MMP9 antibody (MAB936), human MMP-9 biotinylated antibody (BAF911), recombinant human EGFR (1095-ER-002), human EGFR antibody (AF231), human EGFR biotinylated antibody (BAF231), recombinant decorin (143-DE-100), human decorin antibody (BAM1432) and human biotinylated decorin antibody (clone 115413, BAM1431), recombinant human ErbB2 (FC chimera, CF, 1129-ER-050), human ErbB2 antibody (clone 191924, MAB1129), human ErbB2 biotinylated antibody (BAF1129), recombinant human cadherin1 (648-EC-100), human cadherin-1 antibody (clone 77308, MAB18382), and human cadherin-1 biotinylated antibody (BAF648) were derived from R\&D Systems (Minneapolis, MN). Recombinant human IL-6 (14-8069-80), human IL-6 antibody (14-7069-85), and human IL-6 biotinylated antibody (13-7068-85) were purchased from eBioscience (San Diego, CA). The aptamer for EN2 (5'-/5ThioMC6-D/AA AAA AAA AAC GCA TAA TTA CCT CCA GAA GGA GAG GTA ATT ATG CG-3', HPLC purified) [12] was obtained from IDT Integrated DNA Technologies (Coralville, IA). Human EN2 (orb16876) was derived from Biorbyt (Cambridge, UK). Human EN2 biotinylated antibody (bs11552R-biotin) was from BioSS (Woburn, MA). Recombinant human IL-8 (574202), human IL-8 antibody (clone H8A5, 511501), and human IL-8 biotinylated antibody (clone E8N1, 511404) were purchased from Biolegend (San Diego, CA). CRP/Dy647 (CON5, clone 7, D603080) was purchased from Exbio (Vestec, Czech Republic). LowCross-Buffer (100 050) from Candor (Wangen, Germany) was used as assay buffer. Dy647 streptavidin was obtained from Dyomics (Jena, Germany) and Tween 20 and sodium deoxycholate were derived from Sigma-Aldrich (Vienna, Austria). Certified drug-free urine $(88121-\mathrm{CDF}(\mathrm{F}))$ was used from UTAK Laboratories (Valencia, CA).

(2) Chip Fabrication and Processing. Capture antibodies were diluted in spotting buffer $(1 \times \mathrm{PBS}(\mathrm{pH} 7.2) / 0.01 \%$ sodium deoxycholate) to concentrations of $0.4 \mathrm{mg} / \mathrm{mL}$ for MMP-9, decorin, VEGF-A, IL-8, IL-6, MMP-7, cadherin1, ErbB2, and $0.16 \mathrm{mg} / \mathrm{mL}$ for EGFR. The concentration of spotted aptamer EN2 was $100 \mu \mathrm{M}$. The capture elements were printed on proprietary ARChip Epoxy glass slides using the 
Arrayit Nanoprint contact spotter from GeneMachines (pin SMP3). The spot-to-spot distance was $350 \mu \mathrm{m}$. Each probe was spotted in triplicate in 12 identical arrays at a relative humidity of $50 \%$ and kept at $4^{\circ} \mathrm{C}$ for a minimum of three days. The arrayed slides were then blocked for $30 \mathrm{~min}$ in $1 \times$ PBS $(\mathrm{pH} \mathrm{7.2)/0.1 \%} \mathrm{Tween} 20$ and washed twice in $1 \times$ PBS (pH 7.2). For one experiment, a 4-slide set was mounted into an Arrayit Hybridization Cassette $4 \times 16$. The frames create an incubation well for each array $(7 \times 7 \mathrm{~mm})$. We used 12 arrays per slide, resulting in 48 arrays per slide set. The sets accommodate 27 replicate measurements (three arrays with three replicate spots and three repeats) of 10 calibration standards and 45 urine samples. Standard curves for each marker were generated in synthetic urine diluted with assay buffer $(1: 3)$. Each array was incubated with $50 \mu \mathrm{L}$ calibration standard or sample for 2.5 hours. After washing three times with $1 \times$ PBS $(\mathrm{pH} 7.2) / 0.1 \%$ Tween 20 , slides were incubated for $45 \mathrm{~min}$ with $50 \mu \mathrm{L}$ biotinylated antibody mixes $(1 \mu \mathrm{g} / \mathrm{mL})$. Then, the slides were again washed and incubated with $4 \mu \mathrm{g} / \mathrm{mL}$ Dy647 streptavidin. At the end, the slides were washed twice with $1 \times \mathrm{PBS}(\mathrm{pH} 7.2) / 0.1 \%$ Tween 20 and twice in $1 \times$ PBS ( $\mathrm{pH} 7.2)$.

All incubation steps were carried out on the orbital shaker at room temperature. The slides were stored in the dark until scanning.

(3) Data Analysis. Processed slides were scanned using a GenePix 4000B nonconfocal scanner (Molecular Devices, Sunnyvale, CA) $\left(\lambda_{\mathrm{ex}}: 635 \mathrm{~nm}, \lambda_{\mathrm{em}}: 670 \mathrm{~nm}\right)$ and data were analyzed with the GenePix 6.0 software. The photomultiplier tube (PMT) voltage was held constant throughout the scans for each analyte to allow data comparison. The mean signal values were calculated from 27 background corrected data points. Data that were out of the mean signal values \pm the standard deviation (SD) were excluded. Standard curves were set up with GraphPad Prism 5 with a logistic fit.

\subsubsection{Automated Platform for 96-Well Plate ELISA Anal-} ysis. Regarding the second platform, commercially available ELISA kits were used to measure the markers' urinary levels. Supplemental Table 1 (see Supplementary Table 1 in Supplementary Material available online at http://dx.doi.org/10.1155/ 2016/4591910) gives the kits sources and references. The automated analysis was performed using an EVO100 robotic platform (Tecan, Männedorf, Switzerland). Each assay was conducted according to the manufacturer's instructions and included a calibration curve in assay diluent as well as a calibration curve in normal human urine (UTAK Laboratories, Valencia, CA). Both were prepared using protein standards provided in the ELISA kits. Four-parameter logistic regression was used for curve fitting.

\subsection{Data and Statistical Analysis}

2.4.1. Sample Selection. Before the statistical analysis, further sample selection was performed in order to use clinical data most stringent with respect to recurrence events in $\mathrm{BCa}$. A complete history (no missing clinical parameters) is thus required. Based on this additional criterion, 48 of the initial 80 patients were included. Furthermore, three other samples had to be excluded during the biomarker selection process due to lack of valid biomarker readout (see section below) leading to a final study cohort of 45 patients (27 nonrecurrence $\mathrm{BCa}$ patients and 18 recurrence $\mathrm{BCa}$ patients).

2.4.2. Biomarker Selection and Preprocessing. In total, 19 markers were measured in the study. To avoid artifacts caused by missing value imputation, biomarkers were required to have technically valid measurements for all samples in the analysis. Biomarker candidates for the current analysis were selected in such a manner that both the number of included biomarker candidates and the number of included samples are maximized. Under these constraints, a set of 13 biomarker candidates with complete measurements for 45 out of the 48 samples was identified. Ten of the 13 markers were measured with the first platform: decorin, VEGF-A, IL-8, cadherin1, IL-6, EN2, EGFR, ErbB2, MMP-7, and MMP-9. Three were measured with the second platform: IL-8, MMP-9, and fibulin-3.

Measurements below or above the valid range were set to respective lower and upper detection limit values.

2.4.3. Individual Parameter Performance. Descriptive statistics for the two patient groups for the individual clinical parameters and individual biomarker candidates were performed. In addition, their discriminative performance for the two patient groups were evaluated using $Z$ statistic.

2.4.4. Multivariate Statistical Analysis. As the aim of this study was to determine a classifier for recurrence risk, multimarker panels (composed of clinical parameters as well as molecular readouts) were included in multivariable regression models. Two strategies were employed for constructing these models: (i) manual selection based on individual parameter association with the outcome parameter and given clinical evidence and (ii) automatic selection using Least Absolute Shrinkage and Selection Operator (LASSO) regression for determining selection probability of individual parameters.

For the selected parameter sets, a generalized linear model (GLM) for predicting BCa recurrence was constructed on the basis of given sample parameters. Receiver operator characteristics (ROC) along with the correlation matrix for model parameters were determined. Model performance in terms of AUC was also determined. In addition to training AUC values, leave-one-out cross-validation (LOOCV) AUC values were determined.

\section{Results}

3.1. Biomarkers Measurements. Ten markers were analyzed in 1:3 diluted patient's urine using the BCa chip. The assay sensitivity for each marker was visually determined and is displayed in Table 1.

Highest assay sensitivity was observed for decorin (LOD $45 \mathrm{pg} / \mathrm{mL}$ ). Best reproducibility was achieved for markers IL6 and EN2 (CV 7\%). Limits of detection (LOD), linear ranges, 
TABLE 1: Biomarker measurements using the BCa chip.

\begin{tabular}{lcc}
\hline Marker & LOD $(\mathrm{pg} / \mathrm{mL})$ & CV $(\%)$ \\
\hline Decorin & 45 & 11 \\
VEGF-A & 810 & 23 \\
IL-8 & 405 & 9 \\
Cadherin-1 & 2430 & 11 \\
IL-6 & 135 & 7 \\
EN2 & 729 & 7 \\
ErbB2 & 450 & 18 \\
EGFR & 135 & 15 \\
MMP-7 & 810 & 12 \\
MMP-9 & 1350 & 14 \\
\hline
\end{tabular}

LOD: limit of detection.

and $\mathrm{CV}$ of some BCa markers were also described in previous articles ([13] and submitted paper: [14]).

Twelve markers were measured in patient samples using the automated platform for 96-well plate ELISA analysis. The results are summarized in Table 2. No measurement was obtained for uroplakin-3a as the full analysis could not be carried out due to a technical difficulty. All other assays except for fibulin-3 provided a calibration curve in standardized urine. Fibulin-3 concentrations in samples were thus determined using the calibration curve in assay diluent. Three out of the 11 valid assays were not able to detect their specific target in patient urine samples (vimentin, MYC, and p53). Our previous study has shown similar results for vimentin and $\mathrm{p} 53$. Moreover, although MYC was detected in patient samples, the study's ROC analysis ranked MYC as the lowest performing marker [11].

As it was mentioned in Biomarker Selection and Preprocessing, further statistical analysis was only performed on three selected biomarkers: IL-8, MMP-9, and fibulin-3.

Two markers, namely, IL-8 and MMP-9, were measured by both analytical systems. Significant positive correlations were observed for both valid markers with Pearson $R$ values of 0.83 for IL- 8 and 0.70 for MMP-9.

\subsection{Individual Parameters Performance}

3.2.1. Clinical Parameters. In order to evaluate the potential of individual clinical parameters for diagnosing BCa recurrence, these parameters were compared between the two patient groups. Each parameter was also evaluated in terms of discriminative performance for BCa recurrence (Table 3).

No apparent variance is detected in clinical parameters allowing for the prediction of $\mathrm{BCa}$ recurrence. However, even though not significant individually, the parameters no.past.recurrences, BCG.therapy, and no.past.TURBTs appear to exhibit some association with the target parameter. These results clearly indicate the need of including additional molecular biomarkers for improving diagnostic accuracy.

3.2.2. Biomarker Candidates. For each individual biomarker, measurements were compared between the nonrecurrent group and the recurrent group and their discriminative performance is provided in Table 3.
From this analysis, no single biomarker candidate stands out with the desired performance for a recurrence diagnostic test. The highest AUC value, 0.7305, was found for ErbB2. Some markers show AUC values lower than 0.5 (IL-6 ${ }_{\text {chip}}$, MMP-7 ${ }_{\text {chip }}, \mathrm{IL}-8_{\mathrm{AP}}$, and MMP-9 $\left.{ }_{\mathrm{AP}}\right)$. The other markers show AUC values ranging from 0.5247 to 0.6872 . This lack of performance clearly emphasizes the need to use a profile of parameters in order to achieve acceptable diagnostic performance. The most promising markers in terms of individual performance (VEGF-A $A_{\text {chip }}$, IL- $8_{\text {chip }}, E N 2_{\text {chip }}$, and ErbB2 $2_{\text {chip }}$ ) were thus forwarded to multivariate analysis.

3.3. Combined Parameters Performance. As the individual performances of the clinical parameters and the biomarker candidates did not reach a sufficient level, various combinations of both types of parameters were evaluated to define a multiparameter panel. Six multivariate regression models were constructed (see Table 4). The corresponding correlation matrix and the receiver operator characteristics as well as the models' AUC values are provided in Table 4.

Constructed models based on automatically selected parameters show overall superior performance over the models consisting of manually selected parameters. In case of the clinical parameter models (Model 1 and Model 2), the automatically determined model (Model 2) holds one parameter less than the manually selected model but still outperforms the manually constructed model. The drop in the LOOCV AUC of Model 1 may be partially attributed to highly correlated predictor variables no.past.recurrences and no.past.TURBTs. Given the fact that Model 4 holds more parameters than Model 3, the higher training AUC is not surprising. However, Model 4 also exceeds Model 3 in terms of the LOOCV AUC and appears therefore to be more suited for diagnosing BCa recurrence. Similarly, Model 6 comprising parameters from the automatically constructed clinical parameters and biomarker candidates models supersedes its counterpart Model 5. Again, as Model 6 holds one parameter more, the increase in the training AUC (Model 5: 0.82; Model 6: 0.91) was to be expected. Nevertheless, we also observed an increase in the LOOCV AUC (Model 5: 0.64; Model 6: 0.7). Considering all the models presented here, Model 6 shows the best performance in terms of AUC and reaches an AUC of 0.70 in cross-validation.

\section{Discussion}

Due to the high rate of $\mathrm{BCa}$ recurrence, surveillance is a key element of patient management. Thus, great clinical benefit could be gained from improved tools for evidence-based risk assessment, which would influence surveillance strategy. The European Organization for Research and Treatment of Cancer (EORTC) has developed a scoring system to predict short- and long-term risks of disease recurrence and progression after transurethral resection. This scoring system is solely based on conventional clinical and pathological predictors of outcomes (tumor stage and grade, number of tumors, tumor size, concomitant CIS, and history of prior disease recurrence) [15]. EORTC risk tables show significant limitations and its risk classification could be improved by the 
TABLE 2: Biomarker measurements with the automated platform.

\begin{tabular}{|c|c|c|c|c|}
\hline Marker & Calibration curve in assay diluent & Calibration curve in urine & LOD (urine) & Marker detection in samples \\
\hline PTGS2 & - & + & $5 \mathrm{ng} / \mathrm{mL}$ & + \\
\hline FGFR-3 & + & + & $1000 \mathrm{pg} / \mathrm{mL}$ & + \\
\hline Uroplakin-3a & - & - & N/A & N/A \\
\hline Vimentin & + & + & $112.5 \mathrm{ng} / \mathrm{mL}$ & - \\
\hline MYC & + & + & $1.25 \mathrm{ng} / \mathrm{mL}$ & - \\
\hline Tropomodulin-1 & + & + & $2.5 \mathrm{ng} / \mathrm{mL}$ & + \\
\hline BIRC5 & + & + & $1000 \mathrm{pg} / \mathrm{mL}$ & + \\
\hline Fibulin-3 & + & - & $25 \mathrm{ng} / \mathrm{mL}$ (assay diluent) & + \\
\hline p53 & + & + & $10 \mathrm{ng} / \mathrm{mL}$ & - \\
\hline MMP-9 & + & + & $666.7 \mathrm{pg} / \mathrm{mL}$ & + \\
\hline IL-8 & + & + & $125 \mathrm{pg} / \mathrm{mL}$ & + \\
\hline EN2 & + & + & $1.25 \mathrm{ng} / \mathrm{mL}$ & + \\
\hline
\end{tabular}

$+:$ presence of calibration curve or marker detection in samples.

-: absence of calibration curve or no marker detection in samples.

N/A: not applicable; LOD: limit of detection.

TABLE 3: Discriminative performance of individual clinical parameters and biomarker candidates for BCa recurrence.

\begin{tabular}{|c|c|c|c|c|c|}
\hline Clinical parameter & $\operatorname{Pr}(>|z|)$ & AUC & Biomarker candidate & $\operatorname{Pr}(>|z|)$ & AUC \\
\hline diagnosis2sample & 0.42 & 0.50 & Decorin $_{\text {chip }}$ & 0.67 & 0.57 \\
\hline gender & 0.89 & 0.51 & VEGF-A ${ }_{\text {chip }}{ }^{*}$ & 0.05 & 0.67 \\
\hline age.diagnosis & 0.70 & 0.48 & IL- $8_{\text {chip }}{ }^{*}$ & 0.08 & 0.69 \\
\hline age.sample & 0.45 & 0.57 & Cadherin- $1_{\text {chip }}$ & 0.66 & 0.53 \\
\hline grade.diagnosis (G2/G3) & $0.32 / 0.48$ & 0.57 & IL-6 $6_{\text {chip }}$ & 0.65 & 0.48 \\
\hline stage.diagnosis & 0.52 & 0.55 & $\mathrm{EN} 2{ }_{\text {chip }}{ }^{*}$ & 0.09 & 0.65 \\
\hline no.past.recurrences ${ }^{*}$ & 0.08 & 0.63 & EGFR $_{\text {chip }}$ & 0.86 & 0.53 \\
\hline BCG.therapy* $^{*}$ & 0.10 & 0.65 & ErbB2 ${ }_{\text {chip }}{ }^{*}$ & 0.06 & 0.73 \\
\hline mitomycin.therapy & 0.49 & 0.54 & MMP-7 & 0.90 & 0.50 \\
\hline \multirow[t]{4}{*}{ no.past.TURBTs } & 0.33 & 0.58 & MMP-9 $9_{\text {chip }}$ & 0.72 & 0.58 \\
\hline & & & IL- $8_{\mathrm{AP}}$ & 0.74 & 0.47 \\
\hline & & & MMP-9 ${ }_{\mathrm{AP}}$ & 0.46 & 0.50 \\
\hline & & & Fibulin- $3_{\mathrm{AP}}$ & 0.54 & 0.52 \\
\hline
\end{tabular}

* Clinical parameters and biomarker candidates with the best individual AUC.

(a) grade.diagnosis: tumor grade at time of diagnosis; stage.diagnosis: tumor stage at time of diagnosis. The other clinical parameters are defined in the Specimen and Data Collection.

(b) G2/G3: grade 2/grade 3.

(c) Biomarkers ending with chip were measured with the BCa chip and markers ending with AP were measured with the automated platform for 96-well plate ELISA analysis.

updating and addition of new parameters [16-18]. According to a comprehensive review of the literature by Kluth et al. regarding the prognostic and prediction tools in $\mathrm{BCa}$, clinical utility and accuracy of such tools could be improved by biomarkers [17].

Only a few studies have taken into consideration biomarkers as parameters of prediction tools. One specific study identified a panel of 5 cell cycle regulatory biomarkers (cyclin E1, p53, p21, pRB, and p27) which improved the predictive accuracy of $\mathrm{BCa}$ recurrence and survival after cystectomy in patients with pTa-3N0M0 tumors [19]. In another study, Rosser et al. evaluated the performance of a 10-biomarker panel (IL-8, MMP-9, MMP-10, SERPINA1, VEGF-A, ANG, CA9, APOE, SERPINE1, and SDC1) which achieved better sensitivity (79\%) than urine cytology (33\%) for recurrent BCa detection and outperformed any single biomarker [20].
In our discovery study, we evaluated the potential of several biomarkers and clinical parameters for $\mathrm{BCa}$ recurrence diagnosis. Individual parameter performance analysis showed that clinical data alone is not sufficient for the diagnosis of recurrence. Moreover, no single biomarker candidate achieved an acceptable performance by itself. By combining both types of parameters, clinical and molecular, we identified several multivariable regression models. The best performing model (Model 6) achieved an AUC of 0.91, showing better performance than the single parameters. It comprises the following automatically selected (using LASSO) clinical parameters and biomarker candidates: number of past recurrences, number of BCG therapies, stage at time of diagnosis, cadherin-1, IL-8, ErbB2, IL-6, EN2, and VEGF-A.

Regarding clinical parameters, all of the manually selected parameters are also included in Model 6 apart from 
TABLE 4: Multivariate regression models.

\begin{tabular}{lcccc}
\hline Model & Strategy & Description & AUC & AUcluded parameters \\
(LOOCV)
\end{tabular}

(a) Included parameters:

stage.diagnosis: stage of the tumor at time of diagnosis.

The other clinical parameters are defined in the Specimen and Data Collection.

(b) Markers ending with chip were measured with the BCa chip and markers ending with AP were measured with the automated platform for 96-well plate ELISA analysis.

(c) LOOCV: leave-one-out cross-validation.

(d) Biomarker candidates chosen during manual selection for Model 3 are a subset of Model 4 .

age at time of sample and number of TURBTs. The parameter age at time of sample was included during manual selection based on its presumed clinical relevance and was not based on observed statistical association with the target parameter. Number of TURBTs is highly correlated with number of past recurrences and should therefore be omitted.

All the biomarkers identified as relevant in the individual analysis are included in the selected model (IL8, ErbB2, EN2, and VEGF-A). The multivariate analysis provided two additional markers: IL- 6 and cadherin-1. All six biomarkers were previously proved to be of interest with regard to $\mathrm{BCa}$ recurrence. Indeed, a study suggested that assessment of cytoplasmic cadherin-1 staining can predict time to BCa recurrence [21] while Mahnken et al. found a correlation between abnormal cadherin-1 immunostaining and early tumor recurrence, identifying this parameter as an independent predictor of recurrence-free survival [22]. A relationship between high expression levels of IL-8 in malignant tissues and tumor recurrence was also found [23]. A similar finding was described for IL-6 [24]. Moreover, a cytokine panel including both IL- 8 and IL-6 showed potential in tumor recurrence risk identification in patients undergoing BCG treatment [25]. Several studies revealed the correlation between VEGF-A and BCa recurrence, at the mRNA level as well as the protein level [26-28]. As for ErbB2, its overexpression has been associated with $\mathrm{BCa}$ and risks of recurrence and progression but it is not yet clearly established as a molecular marker in recurrent bladder tumors as some studies showed varying results [29-32]. Finally, EN2 has been studied as a potential urinary marker for BCa diagnosis but was also shown to be secreted by recurrent nonmuscle invasive BCa tumors [33].

Although links to recurrence or its mechanism are not clearly established yet for all the selected biomarkers, they all show a potential role for revealing $\mathrm{BCa}$ recurrence. Combining these markers with clinical parameters appears to be a good strategy for achieving acceptable performance with regard to $\mathrm{BCa}$ recurrence diagnosis. Such a panel will allow the determination of patient-specific profiles and could greatly improve recurrence detection.

Although our findings show potential for the development of new BCa surveillance tool, some limitations in our study need to be considered. Indeed, the study includes only a small number of samples due to stringent selection criteria. Furthermore, the study design only allowed evaluating the diagnosis of $\mathrm{BCa}$ recurrence, giving no information on the prediction of BCa recurrence. Validation of our results in an independent cohort shall be warranted.

\section{Conclusions}

In conclusion, cancer is a complex disease and as its detection cannot rely on a single biomarker, the analysis of a profile of biomarkers will likely be more accurate for such purpose [34]. The challenge remains thus in selecting those biomarkers which reflect early tumor growth and disease activity. Our multiplatform strategy allowed the screening of 19 urinary markers for $\mathrm{BCa}$ recurrence diagnosis and led to a selection 
of six markers as well as three clinical parameters defining a panel for patient segmentation. This multiparameter panel outperformed any single biomarker or clinical parameter for $\mathrm{BCa}$ recurrence diagnosis and could be a useful tool for $\mathrm{BCa}$ surveillance scheme.

However, our discovery study shows limitations especially in terms of number of patients. The total number of patient samples included in the statistical analysis was reduced by our selection sample method. Moreover, our study only evaluated the diagnostic aspect of recurrence in nonmuscle invasive BCa patients and did not take into consideration the prediction aspect. According to the IBCN phases classification for the development of diagnostic markers in bladder cancer, our study corresponds to a phase II study, evaluating clinical utility [35]. Further clinical validation of the panel is thus needed, in a phase III study. A larger independent validation study would allow the confirmation of our findings and lead to the design of a novel patient stratification concept as a risk estimating tool. Clinical management of BCa, especially surveillance, could greatly benefit from stratification incorporating patient-specific biomarker and clinical profiles.

\section{Competing Interests}

The authors have declared no competing interests.

\section{Acknowledgments}

This work has been supported in part by the European Commission Program DIPROMON-HEALTH-F5-2012-306157-2: development of an integrated protein- and cell-based device for non-invasive diagnostics in the urogenital tract.

\section{References}

[1] J. Ferlay, I. Soerjomataram, R. Dikshit et al., "Cancer incidence and mortality worldwide: sources, methods and major patterns in GLOBOCAN 2012," International Journal of Cancer, vol. 136, no. 5, pp. E359-E386, 2015.

[2] M. Babjuk, W. Oosterlinck, R. Sylvester et al., "EAU guidelines on non-muscle-invasive urothelial carcinoma of the bladder, the 2011 update," European Urology, vol. 59, no. 6, pp. 997-1008, 2011.

[3] P. E. Clark, N. Agarwal, M. C. Biagioli et al., "Bladder cancer: clinical practice guidelines in oncology," Journal of the National Comprehensive Cancer Network, vol. 11, no. 4, pp. 446-475, 2013.

[4] B. W. G. van Rhijn, M. Burger, Y. Lotan et al., "Recurrence and progression of disease in non-muscle-invasive bladder cancer: from epidemiology to treatment strategy," European Urology, vol. 56, no. 3, pp. 430-442, 2009.

[5] H. Đug, S. Jagodić, J. Ahmetović-Đug, Z. Selimović, and A. Sulejmanović, "Predicting recurrence of non-muscle-invasive bladder cancer after transurethral resection," Medicinski Glasnik, vol. 13, no. 1, pp. 56-61, 2016.

[6] K. D. Sievert, B. Amend, U. Nagele et al., "Economic aspects of bladder cancer: what are the benefits and costs?" World Journal of Urology, vol. 27, no. 3, pp. 295-300, 2009.
[7] R. S. Svatek, B. K. Hollenbeck, S. Holmäng et al., "The economics of bladder cancer: costs and considerations of caring for this disease," European Urology, vol. 66, no. 2, pp. 253-262, 2014.

[8] B. W. G. van Rhijn, H. G. van der Poel, and T. H. van der Kwast, "Urine markers for bladder cancer surveillance: a systematic review," European Urology, vol. 47, no. 6, pp. 736-748, 2005.

[9] J. Parker and P. E. Spiess, "Current and emerging bladder cancer urinary biomarkers," TheScientificWorldJournal, vol. 11, pp. 1103-1112, 2011.

[10] O. P. J. Vrooman and J. A. Witjes, "Urinary markers in bladder cancer," European Urology, vol. 53, no. 5, pp. 909-916, 2008.

[11] M. De Paoli, P. Perco, I. Mühlberger et al., "Disease map-based biomarker selection and pre-validation for bladder cancer diagnostic," Biomarkers, vol. 20, no. 5, pp. 328-337, 2015.

[12] S. Lee, H. Jo, J. Her, H. Y. Lee, and C. Ban, "Ultrasensitive electrochemical detection of engrailed-2 based on homeodomainspecific DNA probe recognition for the diagnosis of prostate cancer," Biosensors and Bioelectronics, vol. 66, pp. 32-38, 2015.

[13] S. Gogalic, U. Sauer, S. Doppler, and C. Preininger, "Bladder cancer biomarker array to detect aberrant levels of proteins in urine," The Analyst, vol. 140, no. 3, pp. 724-735, 2015.

[14] S. Gogalic, U. Sauer, S. Doppler et al., "Validation of a protein panel for the non-invasive detection of recurrent non-muscle invasive bladder cancer," Cancer Epidemiology, Biomarkers \& Prevention, In press.

[15] R. J. Sylvester, A. P. M. van der Meijden, W. Oosterlinck et al., "Predicting recurrence and progression in individual patients with stage Ta T1 bladder cancer using EORTC risk tables: a combined analysis of 2596 patients from seven EORTC trials," European Urology, vol. 49, no. 3, pp. 466-477, 2006.

[16] P. C. Black, "Fine-tuning Risk Stratification for Non-Muscleinvasive Bladder Cancer," European Urology, vol. 69, no. 1, pp. 70-71, 2016.

[17] L. A. Kluth, P. C. Black, B. H. Bochner et al., "Prognostic and prediction tools in bladder cancer: a comprehensive review of the literature," European Urology, vol. 68, no. 2, pp. 238-253, 2015.

[18] W. F. Busato Jr., G. L. Almeida, C. A. Ribas, J. M. Ribas Filho, and O. De Cobelli, "EORTC risk model to predict progression in patients with non-muscle-invasive bladder cancer: is it safe to use in clinical practice?" Clinical Genitourinary Cancer, vol. 14, no. 2, pp. 176-182, 2016.

[19] S. F. Shariat, P. I. Karakiewicz, R. Ashfaq et al., "Multiple biomarkers improve prediction of bladder cancer recurrence and mortality in patients undergoing cystectomy," Cancer, vol. 112, no. 2, pp. 315-325, 2008.

[20] C. J. Rosser, M. Chang, Y. Dai et al., "Urinary protein biomarker panel for the detection of recurrent bladder cancer," Cancer Epidemiology Biomarkers \& Prevention, vol. 23, no. 7, pp. 13401345, 2014.

[21] E. M. Gallagher, D. M. O'Shea, P. Fitzpatrick et al., "Recurrence of urothelial carcinoma of the bladder: a role for insulin-like growth factor-II loss of imprinting and cytoplasmic E-cadherin immunolocalization," Clinical Cancer Research, vol. 14, no. 21, pp. 6829-6838, 2008.

[22] A. Mahnken, I. Kausch, A. C. Feller, and S. Krüger, "E-cadherin immunoreactivity correlates with recurrence and progression of minimally invasive transitional cell carcinomas of the urinary bladder," Oncology Reports, vol. 14, no. 4, pp. 1065-1070, 2005.

[23] S. T. Reis, K. R. M. Leite, L. F. Piovesan et al., "Increased expression of MMP-9 and IL-8 are correlated with poor prognosis of bladder cancer," BMC Urology, vol. 12, article 18, 2012. 
[24] M.-F. Chen, P.-Y. Lin, C.-F. Wu, W.-C. Chen, and C.-T. Wu, "IL-6 Expression regulates tumorigenicity and correlates with prognosis in bladder cancer," PLOS ONE, vol. 8, no. 4, Article ID e61901, 2013.

[25] A. M. Kamat, J. Briggman, D. L. Urbauer et al., "Cytokine Panel for Response to Intravesical Therapy (CyPRIT): nomogram of changes in urinary cytokine levels predicts patient response to Bacillus Calmette-Guérin," European Urology, vol. 69, no. 2, pp. 197-200, 2016.

[26] P. K. Kopparapu, S. A. Boorjian, B. D. Robinson et al., "Expression of VEGF and Its receptors VEGFR1/VEGFR2 is associated with invasiveness of bladder cancer," Anticancer Research, vol. 33, no. 6, pp. 2381-2390, 2013.

[27] J. P. Crew, T. O’Brien, M. Bradburn et al., "Vascular endothelial growth factor is a predictor of relapse and stage progression in superficial bladder cancer," Cancer Research, vol. 57, no. 23, pp. 5281-5285, 1997.

[28] J. P. Crew, T. O’Brien, R. Bicknell, S. Fuggle, D. Cranston, and A. L. Harris, "Urinary vascular endothelial growth factor and its correlation with bladder cancer recurrence rates," The Journal of Urology, vol. 161, no. 3, pp. 799-804, 1999.

[29] P. C.-H. Chen, H.-J. Yu, Y.-H. Chang, and C.-C. Pan, "Her2 amplification distinguishes a subset of non-muscle-invasive bladder cancers with a high risk of progression," Journal of Clinical Pathology, vol. 66, no. 2, pp. 113-119, 2013.

[30] S. Krüger, G. Weitsch, H. Büttner et al., "HER2 overexpression in muscle-invasive urothelial carcinoma of the bladder: prognostic implications," International Journal of Cancer, vol. 102, no. 5, pp. 514-518, 2002.

[31] M. Underwood, J. Bartlett, J. Reeves, D. S. Gardiner, R. Scott, and T. Cooke, "C-erbB-2 gene amplification: a molecular marker in recurrent bladder tumors?" Cancer Research, vol. 55, no. 11, pp. 2422-2430, 1995.

[32] R. A. Gardiner, M. L. T. H. Samaratunga, M. D. Walsh, G. J. Seymour, and M. F. Lavin, "An immunohistological demonstration of c-erbB-2 oncoprotein expression in primary urothelial bladder cancer," Urological Research, vol. 20, no. 2, pp. 117-120, 1992.

[33] R. Morgan, R. T. Bryan, S. Javed et al., "Expression of Engrailed2 (EN2) protein in bladder cancer and its potential utility as a urinary diagnostic biomarker," European Journal of Cancer, vol. 49, no. 9, pp. 2214-2222, 2013.

[34] R. C. Zangar, D. S. Daly, and A. M. White, "ELISA microarray technology as a high-throughput system for cancer biomarker validation," Expert Review of Proteomics, vol. 3, no. 1, pp. 37-44, 2006.

[35] P. J. Goebell, S. L. Groshen, and B. J. Schmitz-Dräger, "Guidelines for development of diagnostic markers in bladder cancer," World Journal of Urology, vol. 26, no. 1, pp. 5-11, 2008. 


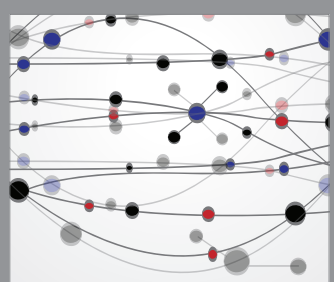

The Scientific World Journal
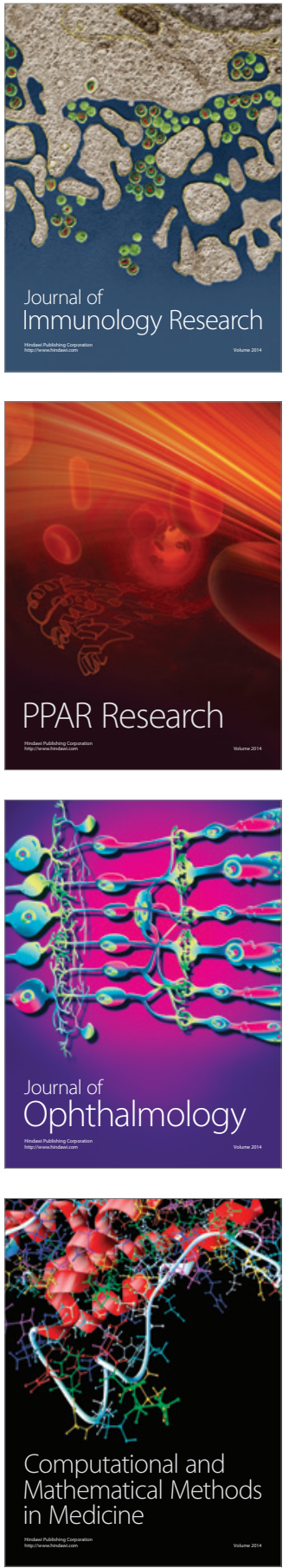

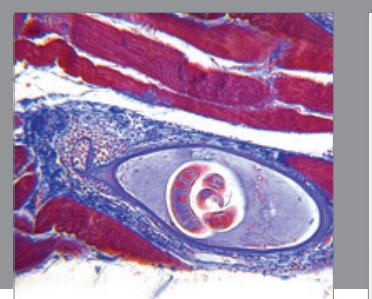

Gastroenterology Research and Practice

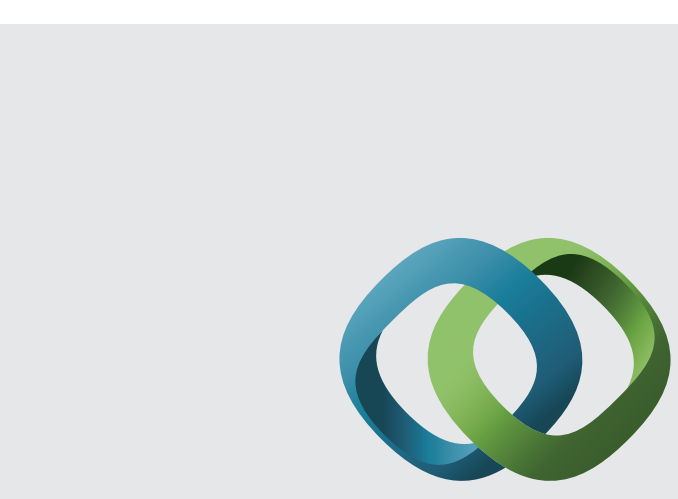

\section{Hindawi}

Submit your manuscripts at

http://www.hindawi.com
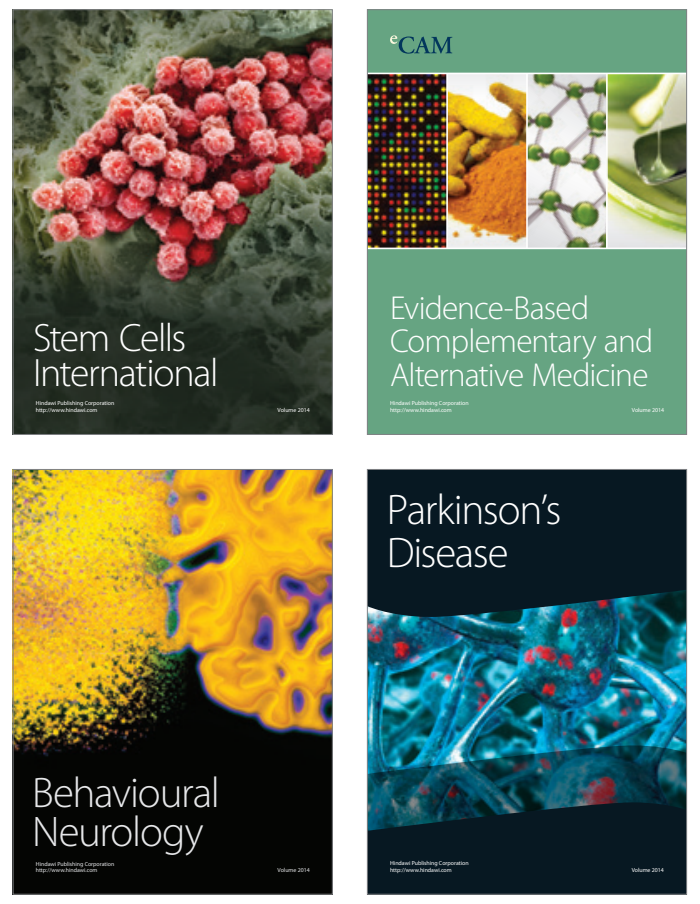
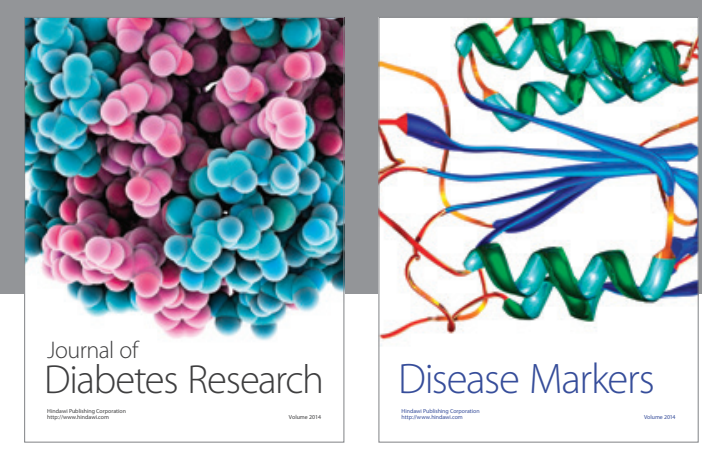

Disease Markers
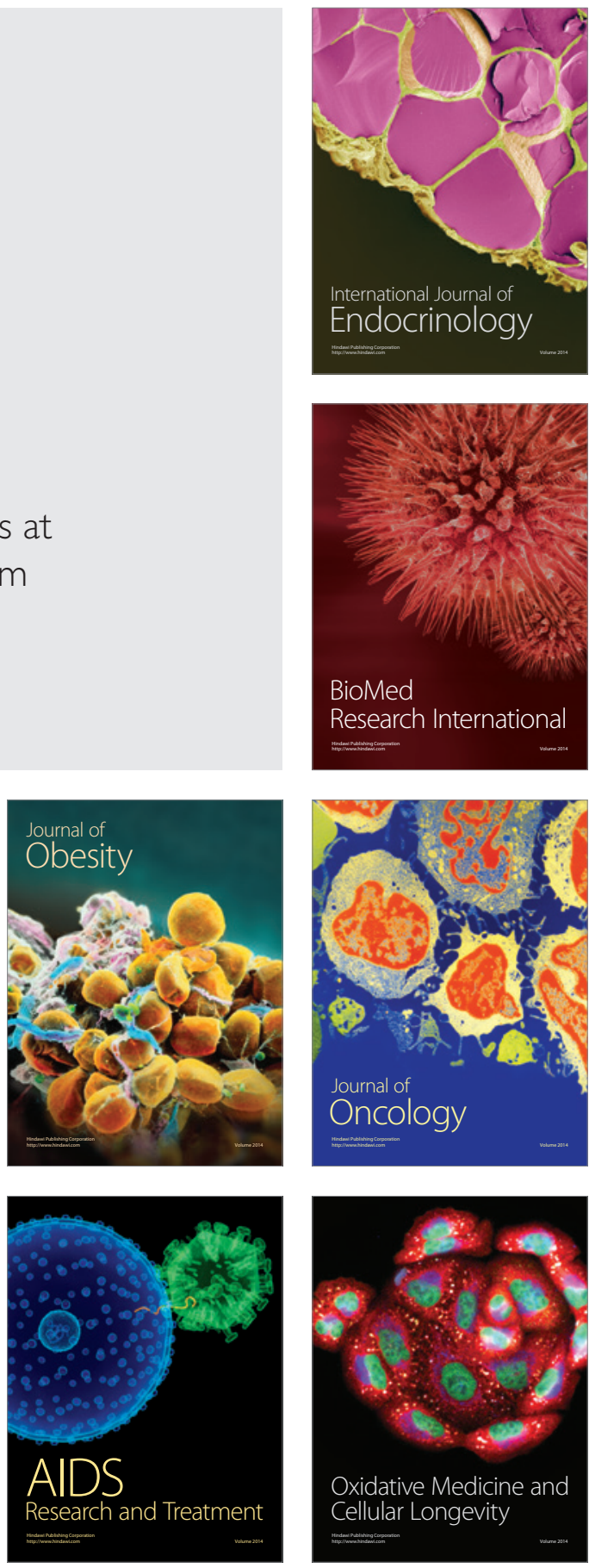